\title{
On modules for double affine Lie algebras
}

\author{
Naihuan Jing and Chunhua Wang*
}

\begin{abstract}
Imaginary Verma modules, parabolic imaginary Verma modules, and Verma modules at level zero for double affine Lie algebras are constructed using three different triangular decompositions. Their relations are investigated, and several results are generalized from the affine Lie algebras. In particular, imaginary highest weight modules, integrable modules, and irreducibility criterion are also studied.
\end{abstract}

\section{Introduction}

Let $\hat{\mathfrak{g}}$ be the untwisted affine Lie algebra associated to a complex finite dimensional simple Lie algebra $\mathfrak{g}$ with Cartan subalgebra $\mathfrak{h}$. The highest weight irreducible $\hat{\mathfrak{g}}$-module $L(\lambda)$ of the highest weight $\lambda$ can be studied with the help of the Verma module $V(\lambda)$, which is an induced module of the one-dimensional module $\mathbb{C} 1_{\lambda}$ of the Borel subalgebra $\hat{\mathfrak{b}}=\hat{\mathfrak{h}}+\hat{\mathfrak{n}}_{+}$such that $\hat{\mathfrak{n}}_{+} 1_{\lambda}=0$. If one partitions the affine root system using the loop realization of $\hat{\mathfrak{g}}$, the associated imaginary Verma module [8] behaves quite differently. For example, the new Verma module can have infinite dimensional weight subspaces.

Double affine Lie algebras are certain central extensions of maps from a 2-dimensional torus to the Lie algebra $\mathfrak{g}$. They are analogous to $\hat{\mathfrak{g}}$ but with two centers, and first appeared in I. Frenkel's work [7] on affinization of Kac-Moody algebras. Moody and Shi [12] have shown that the root systems have different properties from those of the affine root systems. For example, some roots can not be spanned positively or negatively by the "simple" ones. Thus a usual highest weight module would blow up beyond control. In this paper, we generalize imaginary Verma modules (IVM) to double affine Lie algebras and use them to produce irreducible modules.

To study these imaginary Verma modules, we consider generalized imaginary Verma modules, which bare some similarity to the parabolic Verma modules in classical Lie theory. The structure of IVM's is characterized by

2010 Mathematics Subject Classification. Primary: 17B67; Secondary: 17B10, 17B65.

Key words and phrases. double affine Lie algebras, Verma modules, irreducibility, Weyl modules.

*Corresponding author. 
the generalized IVM's, and we show that they are irreducible if the second center is nonzero. When the second center is zero, they are similar to the evaluation modules of the affine Lie algebras, for which we also generalize several results from the Tits-Kantor-Koecher algebras [1].

To further understand the situation of trivial centers, we adopt Chari and Pressley's technique [4, 3] of Weyl modules to modules of double affine Lie algebras, which has played an important role for loop algebras (see 2 for a survey). We remark that the triangular decomposition employed in our case is different from that used in previous work on toroidal Lie algebras (cf. [9, 14, 15]). In our modules, the Borel subalgebras are defined by carving out the second imaginary part to control the growth of the IVM's.

\section{Double affine Lie algebras}

Let $\mathfrak{g}$ be a complex finite dimensional simple Lie algebra of simply laced type with $\mathfrak{h}$ its Cartan subalgebra. Let $\Delta$ be the root system generated by the simple roots $\alpha_{i}(i=1, \ldots, s)$ and let $\alpha_{i}^{\vee} \in \mathfrak{h}(i=1, \ldots, s)$ be the corresponding simple coroots such that $\left\langle\alpha_{j}^{\vee}, \alpha_{i}\right\rangle=a_{i j}$, the entries of the Cartan matrix of $\mathfrak{g}$. Denote by $\theta=\sum_{i=1}^{s} k_{i} \alpha_{i}$ the longest root of $\mathfrak{g}$, and $\theta^{\vee}$ the corresponding dual element in $\mathfrak{h}$. For any positive root $\alpha=\sum_{i} c_{i} \alpha_{i} \in \Delta$, we denote its height by $h t(\alpha)=\sum_{i=1}^{s} c_{i}$.

The double affine Lie algebra $\overline{\mathfrak{T}}$ is the central extension of the 2-loop algebra defined by

$$
\overline{\mathfrak{T}}=\mathfrak{g} \otimes \mathbb{C}\left[t_{1}, t_{1}^{-1}, t_{2}, t_{2}^{-1}\right] \oplus \mathbb{C} c_{1} \oplus \mathbb{C} c_{2},
$$

where $\mathbb{C}\left[t_{1}, t_{1}^{-1}, t_{2}, t_{2}^{-1}\right]$ is the ring of Laurent polynomials in two commuting variables $t_{1}$ and $t_{2}$. Writing $x \otimes t_{1}^{m} t_{2}^{n}$ as $x(m, n)$, the Lie bracket is given by

$$
\begin{aligned}
{\left[x\left(m_{1}, n_{1}\right), y\left(m_{2}, n_{2}\right)\right] } & =[x, y]\left(m_{1}+m_{2}, n_{1}+n_{2}\right) \\
& +\left(x_{1} \mid x_{2}\right) \delta_{m_{1},-n_{1}} \delta_{m_{2},-n_{2}}\left(m_{1} c_{1}+m_{2} c_{2}\right), \\
{\left[c_{1}, x\left(m_{1}, n_{1}\right)\right] } & =\left[c_{2}, x\left(m_{1}, n_{1}\right)\right]=0,
\end{aligned}
$$

where $\left(m_{1}, m_{2}\right),\left(n_{1}, n_{2}\right) \in \mathbb{Z}^{2}, x_{1}, x_{2}, x \in \mathfrak{g}$, and $\left(x_{1} \mid x_{2}\right)$ is the $\mathfrak{g}$-invariant bilinear form. Adjoining the derivations, we define the extended double affine Lie algebra $\mathfrak{T}$ as

$$
\mathfrak{T}=\mathfrak{g} \otimes \mathbb{C}\left[t_{1}, t_{1}^{-1}, t_{2}, t_{2}^{-1}\right] \oplus \mathbb{C} c_{1} \oplus \mathbb{C} c_{2} \oplus \mathbb{C} d_{1} \oplus \mathbb{C} d_{2}
$$

The derivations act on $\mathfrak{T}$ via

$$
\left[d_{i}, x\left(m_{1}, m_{2}\right)\right]=m_{i} x\left(m_{1}, m_{2}\right), \quad\left[d_{i}, c_{j}\right]=0, \quad i, j=1,2 .
$$

Let $\hat{\mathfrak{h}}=\mathfrak{h} \oplus \mathbb{C} c_{1} \oplus \mathbb{C} c_{2} \oplus \mathbb{C} d_{1} \oplus \mathbb{C} d_{2}$ be the Cartan subalgebra of $\mathfrak{T}$, and $\hat{\mathfrak{h}}^{*}$ be the dual space. For $\beta \in \hat{\mathfrak{h}}^{*}$ the root subspace $\mathfrak{T}_{\beta}=\{x \in \mathfrak{T} \mid[h, x]=$ $\beta(h) x, \forall h \in \hat{\mathfrak{h}}\}$ is defined in the usual way. We then define the root system $\Delta_{\mathfrak{T}}$ to be the set of all nonzero $\beta \in \hat{\mathfrak{h}}^{*}$ such that $\mathfrak{T}_{\beta} \neq 0$. It is known that 
12 the root system is different from the usual root system in that a root is no longer a positive or negative sum of the "simple" roots.

To be specific, we let $\delta_{i} \in \hat{\mathfrak{h}}^{*}$ such that $\delta_{i}(\mathfrak{h})=\delta_{i}\left(c_{j}\right)=0$, and $\delta_{i}\left(d_{j}\right)=\delta_{i j}$ for $i, j=1,2$. Then the extended root system

$$
\Delta_{\mathfrak{T}}=\left\{\alpha+\mathbb{Z} \delta_{1}+\mathbb{Z} \delta_{2} \mid \alpha \in \Delta\right\} \cup\left(\left\{\mathbb{Z} \delta_{1}+\mathbb{Z} \delta_{2}\right\} \backslash\{0\}\right),
$$

where the first and second subsets form the real and imaginary roots, denoted by $\Delta_{\mathfrak{T}}^{r e}$ and $\Delta_{\mathfrak{T}}^{i m}$ respectively. The corresponding root subspaces are

$$
\begin{aligned}
\mathfrak{T}_{m \delta_{1}+n \delta_{2}} & =\mathfrak{h} \otimes t_{1}^{m} t_{2}^{n}, \quad(m, n) \neq(0,0), \\
\mathfrak{T}_{\alpha+m \delta_{1}+n \delta_{2}} & =\mathfrak{g}_{\alpha} \otimes t_{1}^{m} t_{2}^{n}, \quad(m, n) \in \mathbb{Z} \times \mathbb{Z} .
\end{aligned}
$$

Then one has the root space decomposition

$$
\mathfrak{T}=\hat{\mathfrak{h}} \oplus \bigoplus_{\beta \in \Delta_{\mathfrak{T}}} \mathfrak{T}_{\beta}
$$

Obviously the extended double affine Lie algebra $\mathfrak{T}$ contains two affine Lie algebras as subalgebras:

$$
\hat{\mathfrak{g}}_{i}=\mathfrak{g} \otimes \mathbb{C}\left[t_{i}, t_{i}^{-1}\right] \oplus \mathbb{C} c_{i} \oplus \mathbb{C} d_{i}, \quad i=1,2 .
$$

Recall that [11] $\alpha_{0}=\delta_{1}-\theta, \alpha_{1}, \ldots, \alpha_{s}$ are the simple roots of $\hat{\mathfrak{g}}_{1}$. Similarly, the roots $\alpha_{-1}=\delta_{2}-\theta, \alpha_{1}, \ldots, \alpha_{s}$ are simple roots for $\hat{\mathfrak{g}}_{2}$. Following [12] we call the elements $\alpha_{-1}, \alpha_{0}, \alpha_{1}, \ldots, \alpha_{s}$ the "simple" roots of $\mathfrak{T}$. However, not all roots can be represented as non-negative or non-positive linear combination of the simple roots. The corresponding coroots are $\alpha_{-1}^{\vee}, \alpha_{0}^{\vee}, \alpha_{1}^{\vee}, \ldots, \alpha_{s}^{\vee}$, where $\alpha_{-1}^{\vee}=c_{2}-\theta^{\vee} \otimes 1$ and $\alpha_{0}^{\vee}=c_{1}-\theta^{\vee} \otimes 1$. Consequently the Cartan subalgebra $\hat{\mathfrak{h}}$ is spanned by $\alpha_{-1}^{\vee}, \alpha_{0}^{\vee}, \alpha_{1}^{\vee}, \ldots, \alpha_{s}^{\vee}, d_{1}, d_{2}$.

\section{Imaginary Verma modules of $\mathfrak{T}$}

Verma modules of affine Lie algebras are defined with the help of a triangular decomposition, which is constructed by a choice of the base in the root system. Since the root system for the extended double affine algebras can not be divided into positive and negative roots in the usual sense, we use a closed subset to partition the root system. For affine Lie algebras Futorny [8] studied a new class of Verma modules called the imaginary Verma modules (IVM) [5, 6] associated with a closed subset defined by a function. For finite dimensional simple Lie algebras, the partition derived from the closed subset coincides with the usual partition of positive and negative roots. In this section, we introduce the imaginary Verma modules for the extended double affine Lie algebras and derive their important properties.

3.1. Imaginary Verma modules. Set $\varphi=\sum_{i=0}^{s} \alpha_{i}^{*}-h t(\theta) \alpha_{-1}^{*}$, where $\alpha_{i}^{*} \in \hat{\mathfrak{h}}$ such that $\alpha_{i}^{*}\left(\alpha_{j}\right)=\delta_{i j}(i, j=-1,0, \ldots, s)$. Clearly, $\varphi\left(\alpha_{i}\right)=1$ for $i=0,1, \ldots, s, \varphi\left(\delta_{2}\right)=0$, and $\varphi\left(\delta_{1}\right)=1+h t(\theta)$. Let $\mathcal{I}=\left\{\alpha \in \Delta_{\mathfrak{T}} \mid \varphi(\alpha)>\right.$ $0\} \cup \mathbb{N} \delta_{2}$. Clearly it is closed under the addition. 
Recall [11] that the root system of $\hat{\mathfrak{g}}_{1}$ is $\Delta_{\hat{\mathfrak{g}}_{1}}=\left\{\alpha+\mathbb{Z} \delta_{1} \mid \alpha \in \Delta\right\} \cup$ $\left(\left\{\mathbb{Z} \delta_{1}\right\} \backslash\{0\}\right)$, and its positive roots are $\Delta_{\hat{\mathfrak{g}}_{1+}}=\left\{ \pm \alpha+\mathbb{N} \delta_{1} \mid \alpha \in \Delta\right\} \cup \Delta_{+} \cup \mathbb{N} \delta_{1}$. Note that for any $\alpha \in \Delta_{\hat{\mathfrak{g}}_{1}+}, \varphi(\alpha)=h t(\alpha)$. Then $\mathcal{I}$ can be written as $\mathcal{I}=$ $\left\{\alpha+\mathbb{Z} \delta_{2} \mid \alpha \in \Delta_{\hat{\mathfrak{g}}_{1}+}\right\} \cup \mathbb{N} \delta_{2}$. Subsequently $\mathcal{I} \cup(-\mathcal{I})=\Delta_{\mathfrak{T}}$ and $\mathcal{I} \cap(-\mathcal{I})=\emptyset$. Denote by $\mathcal{Q}(\mathcal{I})$ the $\mathbb{Z}$-span of $\mathcal{I}$.

Let $\mathfrak{T}_{\mathcal{I}}=\oplus_{\beta \in \mathcal{I}} \mathfrak{T}_{\beta}$ and $\mathfrak{T}_{-\mathcal{I}}=\oplus_{\beta \in-\mathcal{I}} \mathfrak{T}_{\beta}$, then

$$
\mathfrak{T}=\mathfrak{T}_{-\mathcal{I}} \oplus \hat{\mathfrak{h}} \oplus \mathfrak{T}_{\mathcal{I}}
$$

is a triangular decomposition of $\mathfrak{T}$ associated with the closed subset $\mathcal{I}$. The Poincaré-Birkhoff-Witt theorem implies that the universal enveloping algebra

$$
U(\mathfrak{T})=U\left(\mathfrak{T}_{-\mathcal{I}}\right) \otimes U(\hat{\mathfrak{h}}) \otimes U\left(\mathfrak{T}_{\mathcal{I}}\right) .
$$

Let $\mathfrak{b}_{\mathcal{I}}=\hat{\mathfrak{h}} \oplus \mathfrak{T}_{\mathcal{I}}$ be the imaginary Borel subalgebra, which is a solvable Lie algebra. A vector space $V$ is called a weight module of $\mathfrak{T}$ if

$$
V=\bigoplus_{\mu \in \hat{\mathfrak{h}}^{*}} V_{\mu}
$$

where $V_{\mu}=\{v \in V \mid h v=\mu(h) v, \forall h \in \hat{\mathfrak{h}}\}$. Set $\mathcal{P}(V)=\left\{\mu \in \hat{\mathfrak{h}}^{*} \mid V_{\mu} \neq 0\right\}$. We say that $\lambda \geq \mu\left(\lambda, \mu \in \hat{\mathfrak{h}}^{*}\right)$ with respect to $\varphi$ if $\lambda-\mu$ is a non-negative linear combination of roots in $\mathcal{I}$. For simplicity we will omit the reference to $\varphi$ if no confusion arises from the context. For example, both $\mathfrak{T}$ and $U(\mathfrak{T})$ are weight modules for $\mathfrak{T}$.

Definition 3.1. Let $\lambda \in \hat{\mathfrak{h}}^{*}$. A nonzero vector $v$ is called an imaginary highest vector with weight $\lambda$ if $\mathfrak{T}_{\mathcal{I}} \cdot v=0$ and $h \cdot v=\lambda(h) v$ for all $h \in \hat{\mathfrak{h}}$. If $V=U(\mathfrak{T}) v$ then $V$ is called a highest weight module of highest weight $\lambda$.

If $V$ is a highest weight module of weight $\lambda$, then

$$
V=U\left(\mathfrak{T}_{-\mathcal{I}}\right) v=\bigoplus_{\eta \in \mathcal{Q}(\mathcal{I})^{+}} V_{\lambda-\eta}
$$

where $\mathcal{Q}(\mathcal{I})^{+}=\mathbb{Z}_{+}$-span of $\mathcal{I}$.

For $\lambda \in \hat{\mathfrak{h}}^{*}$, let $\mathbb{C} 1_{\lambda}$ be the one-dimensional $\mathfrak{b}_{\mathcal{I}}$-module defined by $(x+$ $h) .1_{\lambda}=\lambda(h) \cdot 1_{\lambda}\left(x \in \mathfrak{T}_{\mathcal{I}}, h \in \hat{\mathfrak{h}}\right)$. The imaginary Verma module is the induced module

$$
\bar{M}(\lambda)=U(\mathfrak{T}) \otimes_{U\left(\mathfrak{b}_{\mathcal{I}}\right)} \mathbb{C} 1_{\lambda} .
$$

3.2. Properties of IVM. Based on the theory of the standard Verma modules [11] and IVM's for the affine Lie algebras [8], the following result can be proved similarly.

Proposition 3.1. For any $\lambda \in \hat{\mathfrak{h}}^{*}$, one has that

(1) $\bar{M}(\lambda)$ is a $U\left(\mathfrak{T}_{-\mathcal{I}}\right)$-free module generated by the imaginary highest vector $1 \otimes 1_{\lambda}$ of weight $\lambda$.

(2) (a) $\operatorname{dim} \bar{M}(\lambda)_{\lambda}=1$;
(b) $0<\operatorname{dim} \bar{M}(\lambda)_{\lambda-k \delta_{2}}<\infty$ for every positive integer $k$; 
(c) If $\bar{M}(\lambda)_{\mu} \neq 0$ and $\mu \neq \lambda-k \delta_{2}$ for any nonnegative integer $k$, then we have $\operatorname{dim} \bar{M}(\lambda)_{\mu}=\infty$.

(3) Any imaginary highest weight $\mathfrak{T}$-module of highest weight $\lambda$ is a quotient of $\bar{M}(\lambda)$.

(4) $\bar{M}(\lambda)$ has a unique maximal submodule $\mathcal{J}$.

(5) If $\mu \in \hat{\mathfrak{h}}^{*}$, then any nonzero homomorphism $\bar{M}(\lambda) \rightarrow \bar{M}(\mu)$ is injective.

We denote by $\bar{L}(\lambda)$ the irreducible quotient $\bar{M}(\lambda) / \mathcal{J}$.

3.3. Irreducibility criterion for IVM. Futorny 8 found that the affine imaginary Verma module is irreducible if and only if the center acts nontrivially. It turns out that a similar result can be obtained for $\bar{M}(\lambda)$ (see Theorem 3.5).

Lemma 3.2. Let $\bar{M}=\bigoplus_{j=0}^{\infty} \bar{M}(\lambda)_{\lambda-j \delta_{2}}$. For any nonzero $v \in \bar{M}(\lambda)$, $U(\mathfrak{T}) v \cap \bar{M} \neq 0$.

Proof. Write $\lambda=\bar{\lambda}-r \delta_{2}$, where $\bar{\lambda}$ is the component of $\Delta_{\hat{\mathfrak{g}}_{1}}$. We can assume that $r$ is minimum so that $v_{\lambda} \neq 0$, otherwise we can replace $\lambda$ by $\lambda^{\prime}$ and $\bar{M}(\lambda)=\bar{M}\left(\lambda^{\prime}\right)$ where $\lambda \equiv \lambda^{\prime} \bmod \left(\mathbb{Z} \delta_{2}\right)$. Therefore $h t_{2}^{-n} v_{\lambda} \neq 0$ for any $n \geq 0$. Assume that $v \in \bar{M}(\lambda)_{\lambda-\mu}$, where $\mu=\sum_{i=0}^{s} n_{i} \alpha_{i}+k \delta_{2}, n_{i} \in \mathbb{Z}_{+}$, and $k \in \mathbb{Z}$. Define the height $h t(\mu)=\sum_{i=0}^{s} n_{i}$ and use induction on $h t(\mu)$. If $h t(\mu)=0$, the weight of $v$ is $\lambda-k \delta_{2}$ for some $k \in \mathbb{Z}_{+}$by assumption, so the result clearly holds.

Let $e_{i}, f_{i}, \alpha_{i}^{\vee}(0 \leq i \leq s)$ be the Chevalley generators of the derived affine Lie algebra $\hat{\mathfrak{g}}_{1}$. If $h t(\mu)>0$, there exists $i_{0} \in\{0, \cdots, s\}$ such that $e_{i_{0}} v \neq 0$. In fact, if $h t(\mu)=1$, say $v=f_{i} t_{2}^{-k} v_{\lambda}$. If $k<0$, then $\alpha_{i}^{\vee} t_{2}^{k-1} v=$ $-2 f_{i} t_{2}^{-1} v_{\lambda} \neq 0$. Then let $v^{\prime}=\alpha_{i}^{\vee} t_{2}^{k-1} v$ and $e_{i} v^{\prime}=-2 \alpha_{i}^{\vee} t_{2}^{-1} v_{\lambda} \neq 0$. The case of $h t(\mu) \geq 2$ is treated similarly. Moreover, $e_{i_{0}}\left(h \otimes t_{2}^{-m}\right) \cdot v^{\prime} \neq 0$ for all $m \geq 0$. Hence $\mathfrak{T}_{\alpha_{i_{0}}-m \delta_{2}} \cdot v^{\prime} \neq 0$, and any of its nonzero element has weight $\lambda-\mu+\alpha_{i_{0}}-(m+1) \delta_{2}$. As $h t\left(\mu-\alpha_{i_{0}}\right)=h t(\mu)-1$, by inductive hypothesis we have $U(\mathfrak{T})\left(\mathfrak{T}_{\alpha_{i_{0}}-m \delta_{2}} \cdot v^{\prime}\right) \cap \bar{M} \neq 0$. Since $U(\mathfrak{T})\left(\mathfrak{T}_{\alpha_{i_{0}}-m \delta_{2}} \cdot v^{\prime}\right) \subset U(\mathfrak{T}) v$, it follows that $U(\mathfrak{T}) v \cap \bar{M} \neq 0$.

Let $\bar{M}(\lambda)^{+}=\left\{v \in \bar{M}(\lambda) \mid \mathfrak{T}_{\mathcal{I}} \cdot v=0\right\}$ be the space of extremal vectors. Clearly $\bar{M}(\lambda)^{+}$is $\hat{\mathfrak{h}}$-invariant. For any nonzero element $v \in \bar{M}(\lambda)^{+}, U(\mathfrak{T}) . v$ generates a submodule of $\bar{M}(\lambda)$. The following result describes the form of extremal vectors.

Corollary 3.3. $\bar{M}(\lambda)^{+} \subset \bar{M}$.

Proof. Suppose on the contrary that there exists a nonzero $v \in \bar{M}(\lambda)^{+} \cap$ $\bar{M}(\lambda)_{\lambda-\mu}$ such that $\mu=\sum_{i=0}^{s} n_{i} \alpha_{i}+k \delta_{2}$ and $h t(\mu)=\sum_{i=0}^{s} n_{i}>0$. Note that 
$U(\mathfrak{T}) v=U\left(\mathfrak{T}_{-\mathcal{I}}\right) v$, so the weight of any homogeneous vector in $U(\mathfrak{T}) v$ is $\lambda-\mu-\nu$ for $\nu \in \mathcal{Q}(\mathcal{I})^{+}$. As $h t(\mu)>0, \lambda-\mu-\nu \neq \lambda\left(\bmod \mathbb{Z} \delta_{2}\right)$. Hence $U(\mathfrak{T}) v \cap \bar{M}=0$, which contradicts with Lemma 3.2 on $\bar{M}(\lambda)_{\lambda-\mu}$.

Define the Heisenberg subalgebra $\hat{\mathfrak{H}}_{2}=\bigoplus_{n \in \mathbb{Z}} \times \mathfrak{h} \otimes t_{2}^{n}+\mathbb{C} c_{2}$, then the space $\bar{M}=\bigoplus_{j=0}^{\infty} \bar{M}(\lambda)_{\lambda-j \delta_{2}}$ is a Verma module for $\hat{\mathfrak{H}}_{2}$. The following result is well-known from Stone-Von Neumann's theorem.

LEMma 3.4. $\bar{M}$ is irreducible as a Verma module for $\hat{\mathfrak{H}}_{2}$ if and only if $\lambda\left(c_{2}\right) \neq 0$.

THEOREM 3.5. The imaginary Verma module $\bar{M}(\lambda)$ is irreducible if and only if $\lambda\left(c_{2}\right) \neq 0$.

Proof. Let $v_{\lambda}$ be the highest weight vector of $\bar{M}(\lambda)$. By definition $\bar{M}(\lambda)$ is irreducible if and only if the space of extremal vectors $\bar{M}(\lambda)^{+}=\mathbb{C} v_{\lambda}$.

Suppose that $\bar{M}(\lambda)$ is irreducible but $\lambda\left(c_{2}\right)=0$. Since $\bar{M}$ is reducible as an $\hat{\mathfrak{H}}_{2}$-module by Lemma 3.4, there exists $w \neq 0$ with weight $\lambda-k \delta_{2}$ $(k>0)$ such that $\mathfrak{T}_{l \delta_{2}} \cdot w=0$ for any $l>0$. It is clear that $\mathfrak{T}_{\beta} \cdot w=0$ for all $\beta \in\left\{\alpha \in \Delta_{\mathfrak{T}} \mid \varphi(\alpha)>0\right\}$, because the weight of $\mathfrak{T}_{\beta} \cdot w$ is larger than $\lambda$. Thus $w \notin \mathbb{C} v_{\lambda}$ is also an imaginary highest vector in $\bar{M}(\lambda)$, which is a contradiction. So we must have $\lambda\left(c_{2}\right) \neq 0$.

If $\lambda\left(c_{2}\right) \neq 0$, Lemma 3.4 implies that the $\hat{\mathfrak{H}}_{2}-$ module $\bar{M}$ is irreducible. Consider any nonzero submodule $U(\mathfrak{T}) v$, where $v \in \bar{M}(\lambda)$. Lemma 3.2 says that $U(\mathfrak{T}) v \cap \bar{M} \neq 0$. Note that $U(\mathfrak{T}) v \cap \bar{M}$ is then a non-trivial $\hat{\mathfrak{H}}_{2^{-}}$ submodule of $\bar{M}$, consequently $U(\mathfrak{T}) v \cap \bar{M}=\bar{M}$, thus $\bar{M} \subset U(\mathfrak{T}) v$. Because $v_{\lambda} \in \bar{M}$, we have that $U(\mathfrak{T}) v=\bar{M}(\lambda)$, i.e., $\bar{M}(\lambda)$ is irreducible.

\section{Generalized IVM $M(\lambda, \mathcal{A})$ and highest weight modules}

In this section, we give a new class of modules $M(\lambda, \mathcal{A})$ generalizing the previous IVM's to study the structure of IVM. They are similar to parabolic Verma modules.

4.1. Definition of $M(\lambda, \mathcal{A})$. Let $\mathcal{A} \subset \mathcal{B}=\{0,1,2, \ldots, s\}$, the index set of $\hat{\mathfrak{g}}_{1}$. We denote $\mathcal{A}^{*}=\mathcal{A} \backslash\{0\}$ and $\mathcal{B}^{*}=\mathcal{B} \backslash\{0\}$. Define $f_{\mathcal{A}}=\sum_{i \in \mathcal{B} \backslash \mathcal{A}} \alpha_{i}^{*}-$ $\left(\sum_{i \in \mathcal{B}^{*} \backslash \mathcal{A}^{*}} k_{i}\right) \alpha_{-1}^{*}$ for $\mathcal{A} \neq \mathcal{B}$ and $f_{\mathcal{B}}=0$, then $f_{\mathcal{A}}\left(\delta_{2}\right)=0$. Set $Q(\mathcal{A})=\{\alpha \in$ $\left.\Delta_{\mathfrak{T}} \mid f_{\mathcal{A}}(\alpha) \geq 0\right\}$ which is closed under addition. Then

$$
Q(\mathcal{A})=\mathcal{I} \cup\left\{-\sum_{i \in \mathcal{A}} l_{i} \alpha_{i}+\mathbb{Z} \delta_{2} \mid l_{i} \geq 0, \prod_{i} l_{i} \neq 0\right\} \cup\left\{-\mathbb{N} \delta_{2}\right\} .
$$

Note that $\mathcal{I} \varsubsetneqq Q(\mathcal{A})$.

The following result is clear.

Proposition 4.1. $Q(\mathcal{A}) \cap(-Q(\mathcal{A}))=\left\{\sum_{i \in \mathcal{A}} \mathbb{Z} \alpha_{i}+\mathbb{Z} \delta_{2}\right\} \cap \Delta_{\mathfrak{T}}$. 
Recall that the Cartan matrix is given by $\left\langle\alpha_{j}^{\vee}, \alpha_{i}\right\rangle=a_{i j}$ for $i=-1,0,1, \ldots, s$, $j=0,1, \ldots, s$. Let $\hat{\mathfrak{h}}_{\mathcal{A}} \subset \hat{\mathfrak{h}}$ be the space spanned by $\alpha_{i}^{\vee}(i \in \mathcal{A})$. Consider the subspaces $\mathfrak{T}_{Q(\mathcal{A})}$ and $\mathfrak{T}_{-\overline{Q(\mathcal{A})}}$, where $\overline{Q(\mathcal{A})}=Q(\mathcal{A}) \backslash(-Q(\mathcal{A}))$. Then $\mathfrak{T}$ decomposes itself as:

$$
\mathfrak{T}=\mathfrak{T}_{-\overline{Q(\mathcal{A})}} \oplus \hat{\mathfrak{h}} \oplus \mathfrak{T}_{Q(\mathcal{A})} .
$$

Let $\lambda \in \hat{\mathfrak{h}}^{*}$ such that $\lambda\left(\hat{\mathfrak{h}}_{\mathcal{A}} \oplus \mathbb{C} c_{2}\right)=0$. Let $\mathbb{C} 1_{\lambda}$ be the one-dimensional $\hat{\mathfrak{h}} \oplus \mathfrak{T}_{Q(\mathcal{A})}$-module such that $(x+h) .1_{\lambda}=\lambda(h) \cdot 1_{\lambda}\left(x \in \mathfrak{T}_{Q(\mathcal{A})}, h \in \hat{\mathfrak{h}}\right)$. Define the induced $\mathfrak{T}$-module associated with $\mathcal{I}, \mathcal{A}$ and $\lambda$ :

$$
M(\lambda, \mathcal{A})=U(\mathfrak{T}) \otimes_{U\left(\hat{\mathfrak{h}} \oplus \mathfrak{T}_{Q(\mathcal{A})}\right)} \mathbb{C} 1_{\lambda}
$$

4.2. Properties of $M(\lambda, \mathcal{A})$. The following result is similar to Proposition 3.1 ,

Proposition 4.2. For any $\lambda \in \hat{\mathfrak{h}}^{*}$ such that $\lambda\left(\hat{\mathfrak{h}}_{\mathcal{A}} \oplus \mathbb{C} c_{2}\right)=0$, one has that

(1) $M(\lambda, \mathcal{A})$ is a $U\left(\mathfrak{T}_{-} \overline{Q(\mathcal{A})}\right)$-free module generated by $1 \otimes 1_{\lambda}$.

(2) $\operatorname{dim} M(\lambda, \mathcal{A})_{\mu}=0,1$ for $\mu=\lambda-\sum_{i \in \mathcal{A}} k_{i} \alpha_{i}-\alpha_{j}+k \delta_{2}, k_{i} \in \mathbb{Z}_{+}$, $k \in \mathbb{Z}$, and $j \in \mathcal{B} \backslash \mathcal{A}$. Otherwise, $\operatorname{dim} M(\lambda, \mathcal{A})_{\mu}=\infty$.

(3) The $\mathfrak{T}$-module $M(\lambda, \mathcal{A})$ has a unique irreducible quotient $L(\lambda, \mathcal{A})$.

(4) Let $\mathcal{A}^{\prime \prime} \subset \mathcal{A}$. Then there exists a chain of surjective homomorphisms

$$
\bar{M}(\lambda) \rightarrow M\left(\lambda, \mathcal{A}^{\prime \prime}\right) \rightarrow M(\lambda, \mathcal{A}) .
$$

(5) Let $\lambda, \mu \in \hat{\mathfrak{h}}^{*}$. Then every nonzero map in $\operatorname{Hom}_{\mathfrak{T}}(M(\lambda, \mathcal{A}), M(\mu, \mathcal{A}))$ is injective.

(6) Let $\mathcal{A} \subset \mathcal{B}$.

Then the module $M(\lambda, \mathcal{A})$ is irreducible if and only if $\lambda\left(\alpha_{i}^{\vee}\right) \neq 0$ for any $i \in \mathcal{A}^{\prime} \backslash \mathcal{A}, \mathcal{A} \varsubsetneqq \mathcal{A}^{\prime}$, i.e. $\mathcal{A}$ is the maximal set such that $\lambda\left(\alpha_{i}^{\vee}\right)=0$.

Remark 4.1. If $\mathcal{A}=\mathcal{B}$, then $M(\lambda, \mathcal{A})=L(\lambda, \mathcal{A})$ is a trivial onedimensional module.

Corollary 4.2. Let $\lambda \in \hat{\mathfrak{h}}^{*}, \mathcal{A} \subset \mathcal{B}$, and $\hat{\mathfrak{h}}_{\mathcal{A}} \oplus \mathbb{C} c_{2} \subset$ ker $\lambda$. Also assume that $\lambda\left(\alpha_{i}^{\vee}\right) \neq 0$ for any $i \in \mathcal{A}^{\prime} \backslash \mathcal{A}, \mathcal{A} \varsubsetneqq \mathcal{A}^{\prime}$. Then $\bar{L}(\lambda) \cong M(\lambda, \mathcal{A})=$ $L(\lambda, \mathcal{A}) \cong L\left(\lambda, \mathcal{A}^{\prime \prime}\right)$ for every $\mathcal{A}^{\prime \prime} \subset \mathcal{A}$.

Proof. Item (6) in Proposition 4.2 implies $M(\lambda, \mathcal{A})=L(\lambda, \mathcal{A})$. Meanwhile, item (4) in Proposition 4.2 implies $L(\lambda, \mathcal{A}) \cong \bar{L}(\lambda)$ and $L\left(\lambda, \mathcal{A}^{\prime \prime}\right) \cong$ $L(\lambda, \mathcal{A})$ for every $\mathcal{A}^{\prime \prime} \subset \mathcal{A}$. This completes the proof.

Corollary 4.3. Let $\lambda \in \hat{\mathfrak{h}}^{*}$ and $\lambda\left(c_{2}\right)=0$. If $\lambda\left(\alpha_{i}^{\vee}\right) \neq 0$ for any $i \in \mathcal{B}$, then $\bar{M}(\lambda)^{+}=\bar{M}$. 
Proof. By Corollary 3.3 , it suffices to show $\bar{M} \subset \bar{M}(\lambda)^{+}$. If $\lambda\left(\alpha_{i}^{\vee}\right) \neq 0$ for any $i \in \mathcal{B}$, then $\bar{L}(\lambda) \cong M(\lambda, \emptyset)=L(\lambda, \emptyset)$. The result follows by comparing the definition of $\bar{L}(\lambda)$ and that of $M(\lambda, \emptyset)$.

The following result is a consequence of Corollary 4.3 ,

TheOREM 4.4. Suppose that $\lambda \in \hat{\mathfrak{h}}^{*}, \lambda\left(c_{2}\right)=0$ and $\lambda\left(\alpha_{i}^{\vee}\right) \neq 0$ for any $i \in \mathcal{B}$. Then one has that

(1) $\bar{M}(\lambda)$ has infinitely many proper submodules:

$$
\bar{M}(\lambda) \supset \bar{M}\left(\lambda-\delta_{2}\right) \supset \bar{M}\left(\lambda-2 \delta_{2}\right) \supset \cdots
$$

where $\operatorname{dim} \bar{M}\left(\lambda-k \delta_{2}\right)_{\lambda-k \delta_{2}}=\operatorname{dim} \bar{M}(\lambda)_{\lambda-k \delta_{2}}=m_{k}$ are finite. Moreover $\bar{L}(\lambda)=\bar{M}(\lambda) / \bar{M}\left(\lambda-\delta_{2}\right)$.

(2) The root multiplicities $\operatorname{dim} \bar{M}(\lambda)_{\lambda-k \delta_{2}}=m_{k}$ for all $k \geq 0$, and $M\left(\lambda-k \delta_{2}, \emptyset\right)$ exhaust all irreducible subquotients of $\bar{M}(\lambda)$.

(3) $\operatorname{dim} H \operatorname{Hom}_{\mathfrak{T}}\left(\bar{M}\left(\lambda-k \delta_{2}\right), \bar{M}(\lambda)\right)=m_{k}$ for any integer $k \geq 0$.

One can describe general highest weight modules as follows.

COROLlary 4.5. Let $V$ be a highest weight $\mathfrak{T}$-module of highest weight $\lambda$. If $c_{2}$ acts trivially and $\lambda\left(\alpha_{i}^{\vee}\right) \neq 0$ for $i=0, \cdots, s$, then $V \simeq \bar{M}(\lambda) / \bar{M}(\lambda-$ $k \delta_{2}$ ) for some $k$.

\section{Highest weight modules of $\mathfrak{T}$}

In this section, we construct another class of highest weight $\mathfrak{T}$-modules by slightly modifying the triangular decomposition for IVM. Using the method of [1], we generalize some results of TKK modules to the highest weight $\mathfrak{T}$ modules under the condition that $\lambda\left(c_{1}\right)=\lambda\left(c_{2}\right)=0$.

These centerless modules are introduced to understand our earlier IVMs and parabolic IVMs. We remark that our construction differs from [14, 15] in that the Cartan subalgebra is purely generated by the imaginary root $\delta_{2}$.

Let $\Phi_{+}=\mathcal{I} \backslash \mathbb{N} \delta_{2}, \Phi_{-}=-\Phi_{+}$, and $\Phi_{0}=\mathbb{Z} \delta_{2}$. Correspondingly, the root spaces are $\mathfrak{T}_{0}=\bigoplus_{\alpha \in \Phi_{0}} \mathfrak{T}_{\alpha}$ and $\mathfrak{T}_{ \pm}=\bigoplus_{\alpha \in \Phi_{+}} \mathfrak{T}_{ \pm \alpha}$. Obviously, $\mathfrak{T}=$ $\mathfrak{T}_{+} \oplus \mathfrak{T}_{-} \oplus \mathfrak{T}_{0}$, and $\Delta_{\mathfrak{T}}=\Phi_{+} \cup \Phi_{-} \cup\left(\Phi_{0} \backslash\{0\}\right)$.

We define a new module structure on $\mathbb{C} 1_{\lambda}$ such that $h .1_{\lambda}=\lambda(h) \cdot 1_{\lambda}(h \in$ $\left.\mathfrak{T}_{0}\right)$, and $\mathfrak{T}_{+} \cdot 1_{\lambda}=0$. Similarly, we define the induced module $M(\lambda)$ of $\mathfrak{T}$ :

$$
M(\lambda)=U(\mathfrak{T}) \otimes_{U\left(\mathfrak{T}_{0} \oplus \mathfrak{T}_{+}\right)} \mathbb{C} 1_{\lambda}
$$

The following result describes the relations among IVM's, generalized IVM's, and highest weight modules.

Proposition 5.1. For any $\lambda \in \hat{\mathfrak{h}}^{*}$, one has that

(1) $M(\lambda)$ is a $U\left(\mathfrak{T}_{-}\right)$-free module generated by $1 \otimes 1_{\lambda}:=v_{\lambda}$.

(2) $M(\lambda)$ has a unique irreducible quotient $L(\lambda)$.

(3) Let $\lambda \in \hat{\mathfrak{h}}^{*}, \hat{\mathfrak{h}}_{\mathcal{A}} \oplus \mathbb{C} c_{2} \subset \operatorname{ker} \lambda$, and $\mathcal{A}^{\prime \prime} \subset \mathcal{A}$. Then there exists a chain of surjective homomorphisms

$$
\bar{M}(\lambda) \rightarrow M(\lambda) \rightarrow M\left(\lambda, \mathcal{A}^{\prime \prime}\right) \rightarrow M(\lambda, \mathcal{A}) .
$$


(4) Let $\mu \in \hat{\mathfrak{h}}^{*}$. Then every nonzero element of $\operatorname{Hom}_{\mathfrak{T}}(M(\lambda), M(\mu))$ is injective.

(5) Let $\mathcal{A} \subset \mathcal{B}$, and $\hat{\mathfrak{h}}_{\mathcal{A}} \oplus \mathbb{C}_{c_{2}} \subset \operatorname{ker} \lambda$. If $\lambda\left(\alpha_{i}^{\vee}\right) \neq 0$ for any $i \in \mathcal{A}^{\prime} \backslash \mathcal{A}$, $\mathcal{A} \varsubsetneqq \mathcal{A}^{\prime}$, then $\bar{L}(\lambda) \cong L(\lambda) \cong L(\lambda, \mathcal{A})$.

REMARK 5.1. (1) If $\mathcal{A}=\emptyset$, then $M(\lambda)=M(\lambda, \mathcal{A})$.

(2) $L(0)$ is one-dimensional.

5.1. Irreducible module $L(\lambda)$. In this subsection, we study integrability of $L(\lambda)$ and its weight subspaces.

Definition 5.2. A $\mathfrak{T}$-module $M$ is called integrable if $M$ is a weight module and $x_{\alpha}(m, n) \in \mathfrak{T}(\alpha \in \Delta ; m, n \in \mathbb{Z})$ are locally nilpotent on every nonzero $v \in M$, i.e., there exists a positive integer $N=N(\alpha, m, n)$ such that $x_{\alpha}(m, n)^{N} . v=0$.

We write $x(\alpha, n)=x_{\alpha} \otimes t_{2}^{n}$ for $\alpha \in \Delta_{\hat{\mathfrak{g}}_{1}}$. The following result is clear.

LEMma 5.3. $\mathfrak{T}_{ \pm}$is generated by $\left\{x\left( \pm \alpha_{i}, n\right) \mid i=0,1, \ldots, s ; n \in \mathbb{Z}\right\}$ respectively.

For an arbitrary Lie algebra $\mathfrak{g}$, we recall the following results.

Proposition 5.2. 11] Let $v_{1}, v_{2}, \ldots$ be a system of generators of a $\mathfrak{g}-$ module $V$, and suppose that each $x \in \mathfrak{g}$ is locally ad-nilpotent on $\mathfrak{g}$ and $x^{N_{i}}\left(v_{i}\right)=0$ for some positive integers $N_{i}(i=1,2, \ldots)$. Then $x$ is locally nilpotent on $V$.

Proposition 5.3. 13 Let $\pi: \mathfrak{g} \rightarrow g l(V)$ be a representation of $\mathfrak{g}$. If both $a d x$ and $\pi(x)$ are locally nilpotent for any $x \in \mathfrak{g}$, then for any $y \in \mathfrak{g}$,

$$
\pi(\exp (a d x)(y))=(\exp \pi(x)) \pi(y)(\exp \pi(x))^{-1} .
$$

For a real root $\gamma \in \Delta_{\mathfrak{\mathfrak { g }}_{1}}^{r e}=\left\{\alpha+n \delta_{1} \mid \alpha \neq 0, n \in \mathbb{Z}\right\}$, define the reflection $r_{\gamma}$ on $\hat{\mathfrak{h}}^{*}$ by

$$
r_{\gamma}(\lambda)=\lambda-\lambda\left(\gamma^{\vee}\right) \gamma
$$

where $\gamma^{\vee}=\alpha^{\vee}+n c_{1}$ if $\gamma=\alpha+n \delta_{1}$. Let $W_{a}$ be the affine Weyl group of $\hat{\mathfrak{g}}_{1}$ generated by the reflections $r_{\gamma}, \gamma \in \Delta_{\mathfrak{g}_{1}}^{r e}$. Then $W_{a}$ is a Coxeter group.

Lemma 5.4. Suppose that $x\left(-\alpha_{i}, n\right)(i=0,1, \ldots, s, n \in \mathbb{Z})$ are locally nilpotent on the highest weight vector $v_{\lambda}$ in $L(\lambda)$. Then all $x(m, n)(m \in \mathbb{Z})$ are locally nilpotent on $L(\lambda)$.

Proof. Since $x\left(-\alpha_{i}, n\right)(i=0,1, \ldots, s)$ are locally nilpotent on $v_{\lambda}$, they act locally nilpotent on any $x(m, n)$ via the adjoint representation. By Prop. 5.2 the elements $x\left(-\alpha_{i}, n\right)$ are locally nilpotent on $L(\lambda)$. So $L(\lambda)$ is integrable for each of the $s l_{2}$-algebras: $\left\langle x\left(\alpha_{i}, n\right), x\left(-\alpha_{i},-n\right), \alpha_{i}^{\vee}\right\rangle$, $i=1,2, \ldots, s$ as well as $\left\langle x\left(\alpha_{0}, n\right), x\left(-\alpha_{0},-n\right), \alpha_{0}^{\vee}=-\theta^{\vee}\right\rangle$ (Note that we assume that $c_{1}, c_{2}$ act as zero).

Suppose $\beta=\alpha+m \delta_{1}+n \delta_{2} \in \Delta_{\mathfrak{T}}^{r e}$ is the root of $x(m, n)$. Let $\gamma=\alpha+m \delta_{1}$, then $\gamma=\beta-n \delta_{2} \in \Delta_{\mathfrak{g}_{1}}^{r e}$. For any $i \in\{0, \ldots, s\}$, there exists a $w \in W_{a}$ such 
that $w(\gamma)=\alpha_{i}$ [11. Since $w\left(\delta_{2}\right)=\delta_{2}$, we have $w(\beta)=\alpha_{i}+n \delta_{2}$. Let $s_{w}$ be the linear automorphism of $\mathfrak{T}$ associated with $w$. Up to a nonzero constant, we have $s_{w}(x(m, n))=Y$ for $Y \in \mathfrak{T}_{\alpha_{i}+n \delta_{2}}$. It follows from Prop. 5.3 that all $x(m, n)$ are locally nilpotent on $L(\lambda)$.

We now recall Weyl modules [4] for the loop algebra $\hat{\mathfrak{s l}}_{2}(\mathbb{C})=\mathfrak{s l}_{2} \otimes$ $\mathbb{C}\left[t, t^{-1}\right]$. Let $a_{1}, \ldots, a_{n} \in \mathbb{C}^{\times}$and $\lambda_{1}, \ldots, \lambda_{n} \in \mathbb{Z}_{+}$with $|\lambda|=\sum_{i} \lambda_{i}$. We define $B(a, \lambda)$ to be the cyclic $\hat{\mathfrak{s l}}_{2}(\mathbb{C})$-module generated by $w$ such that

$$
\begin{aligned}
& e(m) w=f(0)^{|\lambda|+1} w=0, \quad \forall m, \\
& h(m) w=\sum_{j=1}^{n} \lambda_{j} a_{j}^{m} w, \quad \forall m .
\end{aligned}
$$

The following result was proved by Chari and Pressley.

Proposition 5.4. [4] The $\hat{\mathfrak{s l}}_{2}(\mathbb{C})$-module $B(a, \lambda)$ is finite dimensional. If $B^{\prime}$ is a finite dimensional $\hat{\mathfrak{s l}}_{2}(\mathbb{C})$-module generated by $w^{\prime}$ such that $\operatorname{dim} U\left(\alpha^{\vee} \otimes\right.$ $\left.\mathbb{C}\left[t, t^{-1}\right]\right) w^{\prime}=1$, then $B^{\prime}$ is a quotient of some $B(a, \lambda)$ constructed above.

We also need the following remarkable formula proved by Garland.

Lemma 5.5. [10] Let $\beta=\alpha+r_{1} \delta_{1} \in \Delta_{\hat{\mathfrak{g}}_{1}+}, r_{1} \in \mathbb{Z}$. Then for any $t \geq 1$, we have

$$
\begin{aligned}
x(\beta, \pm 1)^{t} x(-\beta, 0)^{t+1} & =\sum_{m=0}^{t} x(-\beta, \pm m) \Lambda^{ \pm}\left(\beta^{\vee}, t-m\right)+X, \\
x(\beta, \pm 1)^{t+1} x(-\beta, 0)^{t+1} & =\Lambda^{ \pm}\left(\beta^{\vee}, t+1\right)+Y,
\end{aligned}
$$

where $X$ and $Y$ are in the left ideal of $\mathfrak{T}$ generated by the subalgebra $\mathfrak{T}_{+}$and $\Lambda^{ \pm}\left(\beta^{\vee}, j\right)$ is the coefficient of $u^{j}$ in $\Lambda^{ \pm}\left(\beta^{\vee}, u\right)=\exp \left(-\sum_{j=1}^{\infty} \frac{\beta^{\vee} t_{2}^{ \pm j} u^{j}}{j}\right)$.

REMARK 5.6. By definition of $\Lambda^{ \pm}\left(\beta^{\vee}, u\right)$ it follows that every element $h \otimes t_{2}^{m}\left(h \in \mathfrak{h}, m \in \mathbb{Z}^{\times}\right)$is a polynomial in the variables $\Lambda^{ \pm}\left(\alpha_{i}^{\vee}, j\right)(i=$ $1,2, \ldots, s ; j \in \mathbb{N})$.

TheOREM 5.7. For each $p=1, \ldots, s$, let $\lambda_{p, i} \in \mathbb{Z}_{+}, a_{p, i} \in \mathbb{C}^{\times}, i=$ $1, \cdots, k_{p}$. If $\lambda$ satisfies

$$
\lambda\left(\alpha_{p}^{\vee}(0, n)\right)=\sum_{i=1}^{k_{p}} \lambda_{p, i} a_{p, i}^{n}
$$

and $\lambda\left(c_{1}\right)=\lambda\left(c_{2}\right)=0$, then

(1) $L(\lambda)$ is integrable.

(2) $L(\lambda)=U\left(\hat{\mathfrak{n}}_{1-} \otimes \mathbb{C}\left[t_{2}\right]\right) \cdot v_{\lambda}$, where $\hat{\mathfrak{n}}_{1-}$ is the negative nilpotent subalgebra of $\hat{\mathfrak{g}}_{1}$.

Moreover if $L(\lambda)$ and $L\left(\lambda^{\prime}\right)$ are nonzero irreducible modules, then

$$
L(\lambda) \cong L\left(\lambda^{\prime}\right) \Longleftrightarrow \lambda=\lambda^{\prime} .
$$


Proof. (1) By Lemma 5.4 it suffices to show that $x\left(-\alpha_{i}, n\right)$ are locally nilpotent on $L(\lambda)$ for $i=0,1, \ldots, s$ and $n \in \mathbb{Z}$. Since $L(\lambda)$ is irreducible, one only needs to prove that there exist $N \geq 0$ such that

$$
x\left(\alpha_{j}, m\right) x\left(-\alpha_{i}, n\right)^{N} \cdot v_{\lambda}=0(i, j=0,1, \ldots, s ; m, n \in \mathbb{Z}) .
$$

If $j \neq i$, then $\alpha_{j}-\alpha_{i}+(m+n) \delta_{2}$ is not a root. So (5.4) holds.

If $j=i$, denote $x_{n}=x\left(\alpha_{i}, n\right), y_{n}=x\left(-\alpha_{i}, n\right), h_{n}=\alpha_{i}^{\vee}(0, n)$. Then we have

$$
\left[x_{m}, y_{n}\right]=h_{m+n},\left[h_{p}, x_{m}\right]=2 x_{p+m},\left[h_{p}, y_{n}\right]=-2 y_{p+n} .
$$

So $\left\{x_{n}, y_{n}, h_{n}: n \in \mathbb{Z}\right\}$ is a basis of the loop algebra $\hat{\mathfrak{s l}}_{2}(\mathbb{C})$. We consider the subspace $U\left(\hat{\mathfrak{s l}}_{2}(\mathbb{C})\right) v_{\lambda}$ inside $M(\lambda)$. It follows from Prop. 5.4 that $x\left(-\alpha_{i}, n\right)^{N} v_{\lambda}$ belongs to a proper submodule of $U\left(\hat{\mathfrak{s l}}_{2}(\mathbb{C})\right) v_{\lambda}$ for some $N \geq 0$. In fact, if $x\left(-\alpha_{i}, n\right)^{N} v_{\lambda}$ does not belong to the proper maximal submodule $M$ of $U\left(\hat{\mathfrak{s}}_{2}(\mathbb{C})\right) v_{\lambda}$ for any $N \geq 0$, then each $x\left(-\alpha_{i}, n\right)^{N} v_{\lambda}+M(N \in \mathbb{N})$ is non-zero in the irreducible quotient $U^{\prime}=U\left(\hat{\mathfrak{s l}}_{2}(\mathbb{C})\right) v_{\lambda} / M$. Therefore $U^{\prime}$ is infinite dimensional, but it is also isomorphic to some $B(a, \lambda)$. This is a contradiction by Prop. 5.4. Applying PBW theorem to $M(\lambda)$, we get Eq. (5.4), which finishes the proof.

(2) We consider the action of real root vectors $x\left(-\alpha_{i},-r\right)$, where $i=$ $0,1, \ldots, s$ and $r \in \mathbb{Z}_{+}$. By (1), there exists a (minimal) positive integer $N_{i}$ such that

$$
x\left(-\alpha_{i}, 0\right)^{N_{i}+1} v_{\lambda}=0 .
$$

Let $x\left(\alpha_{i}, 1\right)^{N_{i}}$ act on Eq.(5.5) and by Lemma 5.5, we get

$$
\sum_{m=0}^{N_{i}} x\left(-\alpha_{i}, m\right) \Lambda^{+}\left(\alpha_{i}^{\vee}, N_{i}-m\right) \cdot v_{\lambda}=0 .
$$

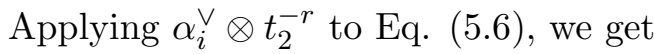

$$
\sum_{m=0}^{N_{i}} x\left(-\alpha_{i}, m-r\right) \Lambda^{+}\left(\alpha_{i}^{\vee}, N_{i}-m\right) \cdot v_{\lambda}=0 .
$$

Therefore $x\left(-\alpha_{i},-r\right) \cdot v_{\lambda}$ is written as a linear combination of the elements in the set $\left\{x\left(-\alpha_{i}, m\right) \cdot v_{\lambda}, m>-r\right\}$. We claim that $\Lambda^{+}\left(\alpha_{i}^{\vee}, N_{i}\right) \cdot v_{\lambda} \neq 0$. In fact, applying $\alpha_{i}^{\vee}(0,-1)$ to Eq.(5.5), one gets that $x\left(-\alpha_{i},-1\right) x\left(-\alpha_{i}, 0\right)^{N_{i}} v_{\lambda}=0$. Note that the subalgebra $\left\{x\left(\alpha_{i}, 1\right), x\left(-\alpha_{i},-1\right), \alpha_{i}^{\vee}\right\}$ is isomorphic to $s l_{2}$, then $x\left(\alpha_{i}, 1\right)^{q} x\left(-\alpha_{i}, 0\right)^{N_{i}} v_{\lambda} \neq 0\left(0 \leq q \leq N_{i}\right)$ by properties of $s l_{2}$-modules. When $N_{i}=0, \Lambda^{+}\left(\alpha_{i}^{\vee}, 0\right)=1$. If $N_{i}=1$, note that $x\left(\alpha_{i}, 1\right) x\left(-\alpha_{i}, 0\right) v_{\lambda} \neq 0$, so $\Lambda^{+}\left(\alpha_{i}^{\vee}, 1\right) \cdot v_{\lambda}=-\alpha_{i}^{\vee}(0, i) \cdot v_{\lambda} \neq 0$. If $N_{i}>1$, we choose $q=N_{i}$ in the previous equation. Then Lemma 5.5 implies that $\Lambda^{+}\left(\alpha_{i}^{\vee}, N_{i}\right) \cdot v_{\lambda} \neq 0$. Using induction on $r$, one shows that for arbitrary $r>0$, the element $x\left(-\alpha_{i},-r\right) \cdot v_{\lambda}$ can be represented by the elements of the form $\left\{x\left(-\alpha_{i}, m\right) \cdot v_{\lambda}, m \geq 0\right\}$. This completes the proof by Lemma 5.3. The last statement is easily seen. 
The IVM's have both finite and infinite dimensional weight subspaces. In the following, we study the weight spaces of an irreducible $L(\lambda)$ as a module for $\hat{\mathfrak{h}}^{\prime}=\mathfrak{h} \oplus \mathbb{C} c_{1} \oplus \mathbb{C} c_{2} \oplus \mathbb{C} d_{1}$.

Let $\mathfrak{T}^{\prime}=\mathfrak{g} \otimes \mathbb{C}\left[t_{1}^{ \pm 1}, t_{2}^{ \pm 1}\right] \oplus \mathbb{C} c_{1} \oplus \mathbb{C} c_{2} \oplus \mathbb{C} d_{1}$. Set $\hat{\mathfrak{h}}^{\prime}\left[t_{2}^{ \pm}\right]=\operatorname{span}\left\{\mathfrak{h}(0, n), c_{1}\right.$, $\left.c_{2}, d_{1} ; n \in \mathbb{Z}\right\}$, which is abelian. Note that $\mathfrak{T}_{0}=\hat{\mathfrak{h}}^{\prime}\left[t_{2}^{ \pm}\right] \oplus \mathbb{C} d_{2}$. Hence $\mathfrak{T}^{\prime}=\mathfrak{T}_{+} \oplus \mathfrak{T}_{-} \oplus \hat{\mathfrak{h}}^{\prime}\left[t_{2}^{ \pm}\right]$.

THEOREM 5.8. If $\lambda$ satisfies the conditions of Theorem 5.7 (cf. (5.2)), then the weight spaces of $L(\lambda)$ are finite dimensional as $\mathfrak{T}^{\prime}$-modules with respect to $\hat{\mathfrak{h}}^{\prime}=\mathfrak{h} \oplus \mathbb{C} c_{1} \oplus \mathbb{C} c_{2} \oplus \mathbb{C} d_{1}$.

Proof. Let $\left.\lambda\right|_{\hat{\mathfrak{h}}^{\prime}}=\lambda_{1}$. Since $d_{2}$ is removed, the root $\delta_{2}$ can be viewed as nullified, thus the weight set $P(L(\lambda)) \subset \lambda_{1}-\left(\sum_{i=0}^{s} \mathbb{Z}_{+} \alpha_{i}\right)$. Consider the weight space $L(\lambda)_{\lambda_{1}-\varepsilon}$, where $\varepsilon \in \sum_{i=0}^{s} \mathbb{Z}_{+} \alpha_{i}$. By PBW theorem, $L(\lambda)_{\lambda_{1}-\varepsilon}$ is spanned by

$$
x\left(\beta_{1}, n_{1}\right) x\left(\beta_{2}, n_{2}\right) \cdots x\left(\beta_{k}, n_{k}\right) \cdot v_{\lambda},
$$

where $\beta_{1}, \ldots, \beta_{k}$ are negative roots of the affine Lie algebra $\hat{\mathfrak{g}}_{1}$ such that $\varepsilon=-\sum_{i=0}^{k} \beta_{i}$ and $n_{i} \in \mathbb{Z}$. There are only finitely many $\beta_{i}$ for a given $\varepsilon$.

For fixed $\beta_{i}$ 's, $x\left(\beta_{1}, n_{1}\right) \cdots x\left(\beta_{k}, n_{k}\right) . v_{\lambda}\left(n_{i} \in \mathbb{Z}\right)$ generate a finite dimensional subspace. In fact, define $e_{p}\left(t_{2}\right)=\prod_{j=1}^{k_{p}}\left(t_{2}-a_{p, j}\right)=\sum_{i=0}^{k_{p}} \epsilon_{p, i} t_{2}^{i}$. Let $E_{p}=e_{p}\left(t_{2}\right) \mathbb{C}\left[t_{2}, t_{2}^{-1}\right]$. By $e_{p}\left(a_{p j}\right)=0$, it is easy to check that

$$
\lambda\left(\alpha_{p}^{\vee} \otimes E_{p}\right)=0, p=1,2, \ldots, s .
$$

Let $e\left(t_{2}\right)=\prod_{p=1}^{s} e_{p}\left(t_{2}\right)=\sum_{i=0}^{k} \epsilon_{i} t_{2}^{i}$, where $k=\sum_{i=1}^{p} k_{i}$. Set $E=e\left(t_{2}\right) \mathbb{C}\left[t_{2}, t_{2}^{-1}\right] \subset$ $E_{p}$, then

$$
\lambda\left(\alpha_{p}^{\vee} \otimes E\right)=0 .
$$

First we show for any negative affine root $\beta$ of $\hat{\mathfrak{g}}_{1}$

$$
\sum_{i=0}^{k} \epsilon_{i} x(\beta, m+i) \cdot v_{\lambda}=0, \forall m \in \mathbb{Z}
$$

in $L(\lambda)$. Since $L(\lambda)$ is irreducible, it is enough to check that $\mathfrak{T}_{+}$annihilates the left-hand side of (5.10). By Lemma 5.3 this means that $x\left(\alpha_{j}, n\right)$ kills the LHS of (5.10) for any $j \in\{0, \ldots, s\}$. We use induction on $h t(-\beta)$.

First of all, let us consider the case of $h t(-\beta)=1$ say $\beta=-\alpha_{p}$. If $p \neq j$, then clearly $x\left(\alpha_{j}, n\right)$ annihilates the LHS of (5.10). Also

$$
x\left(\alpha_{p}, n\right)\left(\sum_{i=0}^{k} \epsilon_{i} x\left(-\alpha_{p}, m+i\right) \cdot v_{\lambda}\right)=\sum_{i=0}^{k} \epsilon_{i} \alpha_{p}^{\vee}(0, m+n+i) \cdot v_{\lambda}=0
$$


by (5.9), where $\alpha_{0}^{\vee}=-\theta^{\vee}$ as $c_{1}$ acts as 0 . Hence Eq. (5.10) holds for $h t(-\beta)=1$.

Now consider general $\beta$ of $h t(-\beta)>1$. There exists a simple root $\alpha_{j}$ such that $\alpha_{j}+\beta$ is a negative affine root and $h t\left(-\alpha_{j}-\beta\right)<h t(-\beta)$. Therefore

$$
x\left(\alpha_{j}, n\right)\left(\sum_{i=0}^{k} \epsilon_{i} x(\beta, m+i) \cdot v_{\lambda}\right)=\sum_{i=0}^{k} \epsilon_{i} x\left(\alpha_{j}+\beta, n+m+i\right) \cdot v_{\lambda}=0
$$

by the induction hypothesis.

Next we show that

$$
\sum_{i=0}^{k} \epsilon_{i} x\left(\gamma_{1}, n_{1}\right) \cdots x\left(\gamma_{j}, m+i\right) x\left(\gamma_{j+1}, n_{j+1}\right) \cdots x\left(\gamma_{l}, n_{l}\right) \cdot v_{\lambda}=0
$$

for any fixed $\gamma_{1}, \cdots, \gamma_{l}$ in $\Delta_{\hat{\mathfrak{g}}_{1}-}$. This is again proved by another induction on $h t\left(-\gamma_{j+1}-\cdots-\gamma_{l}\right)$.

If the height of $-\left(\gamma_{j+1}+\cdots+\gamma_{l}\right)$ is 0 , Eq. (5.11) is clear. Then

$$
\begin{array}{r}
\sum_{i=0}^{k} \epsilon_{i} x\left(\gamma_{1}, n_{1}\right) \cdots x\left(\gamma_{j}, m+i\right) x\left(\gamma_{j+1}, n_{j+1}\right) \cdots x\left(\gamma_{l}, n_{l}\right) \cdot v_{\lambda} \\
=\sum_{i=0}^{k} \epsilon_{i} x\left(\gamma_{1}, n_{1}\right) \cdots\left[x\left(\gamma_{j}, m+i\right), x\left(\gamma_{j+1}, n_{j+1}\right)\right] \cdots x\left(\gamma_{l}, n_{l}\right) \cdot v_{\lambda}+ \\
\sum_{i=0}^{k} \epsilon_{i} x\left(\gamma_{1}, n_{1}\right) \cdots x\left(\gamma_{j+1}, n_{j+1}\right) x\left(\gamma_{j}, m+i\right) \cdots x\left(\gamma_{l}, n_{l}\right) \cdot v_{\lambda} .
\end{array}
$$

Each term of the right hand side is zero by induction hypothesis. Therefore Eq. (5.11) holds.

For fixed $\beta_{1}, \beta_{2}, \ldots, \beta_{k}$, the vectors of the form (5.8) generate a finite dimensional weight space due to the fact that $\operatorname{dim} \mathbb{C}\left[t_{2}, t_{2}^{-1}\right] / E<\infty$. This finishes the proof.

THEOREM 5.9. If $L(\lambda)$ is irreducible as a $\mathfrak{T}^{\prime}$-module with finite dimensional weight spaces and the action of $c_{1}$ and $c_{2}$ are zero, then $\lambda$ satisfies the conditions of Theorem 5.7 (cf. (5.2)).

Proof. For $p \neq 0$, the algebra $\mathfrak{L}$ generated by $\left\{x_{\alpha_{p}}, x_{-\alpha_{p}}, \alpha_{p}^{\vee}\right\}$ is isomorphic to $\mathfrak{s l}_{2}$. Let $V$ be the irreducible quotient of $U(\hat{\mathfrak{L}}) \cdot v_{\lambda}$, where $\hat{\mathfrak{L}}=$ $\mathfrak{L} \otimes \mathbb{C}\left[t_{2}, t_{2}^{-1}\right] \oplus \mathbb{C} c_{2}$. Since the weight spaces of $L(\lambda)$ are finite dimensional, the set $\left\{x\left(-\alpha_{p}, n\right) \cdot v_{\lambda}, n \in \mathbb{Z}\right\}$ is linearly dependent. Thus there exists a nonzero polynomial $g=\sum_{i} g_{i} t_{2}^{i}$ such that $x_{-\alpha_{p}} \otimes g \cdot v_{\lambda}=0$. Let $G\left(t_{2}\right)=g \mathbb{C}\left[t_{2}, t_{2}^{-1}\right]$, then $x_{-\alpha_{p}} \otimes G \cdot v_{\lambda}=0$. In fact,

$$
0=\left(\alpha_{p}^{\vee} \otimes t_{2}^{m}\right) x_{-\alpha_{p}} \otimes g \cdot v_{\lambda}=\left(x_{-\alpha_{p}} \otimes g\right) \alpha_{p}^{\vee} \otimes t_{2}^{m} \cdot v_{\lambda}-2 x_{-\alpha_{p}} \otimes t_{2}^{m} g \cdot v_{\lambda}
$$

and $\alpha_{p}^{\vee} \otimes t_{2}^{m} . v_{\lambda}=\lambda\left(\alpha_{p}^{\vee} \otimes t_{2}^{m}\right) . v_{\lambda}$. Naturally, $\alpha_{p}^{\vee} \otimes G . v_{\lambda}=0$. Subsequently $\left(\mathfrak{L} \otimes G \oplus \mathbb{C} c_{2}\right) \cdot v_{\lambda}=0$. 
Set $W=\left\{v \in V \mid\left(\mathfrak{L} \otimes G \oplus \mathbb{C} c_{2}\right) \cdot v=0\right\}$. Clearly $W$ is a nonzero submodule of $V$. Thus $V=W$ due to irreducibility of $V$. Then $V$ is a $\hat{\mathfrak{L}} / \mathfrak{L} \otimes G \oplus$ $\mathbb{C} c_{2}-$ module, thus $\operatorname{dim} V<\infty$. By Prop. 5.4, $\lambda$ satisfies the conditions of Theorem 5.7 .

REMARK 5.10. The above proof also shows that when $c_{1}$ and $c_{2}$ act trivially, the irreducible $\mathfrak{T}^{\prime}$-module $L(\lambda)$ has finite dimensional weight spaces if and only if there is an ideal $\mathcal{S}$ of $\mathbb{C}\left[t_{2}, t_{2}^{-1}\right]$ such that $\lambda\left(\alpha_{p}^{\vee} \otimes \mathcal{S}\right)=0$, $p=1,2, \ldots, s$.

Corollary 5.11. Let $L(\lambda)$ be an irreducible $\mathfrak{T}^{\prime}$-module with finite dimensional weight spaces and suppose that the centers $c_{1}$ and $c_{2}$ act trivially. Then there exists an ideal $\mathcal{S}$ of $\mathbb{C}\left[t_{2}, t_{2}^{-1}\right]$ such that $\widetilde{\mathfrak{g}}_{1} \otimes \mathcal{S} . L(\lambda)=0$, where $\widetilde{\mathfrak{g}}_{1}=\mathfrak{g} \otimes \mathbb{C}\left[t_{1}, t_{1}^{-1}\right]$.

Proof. First there exists an ideal $\mathcal{S}$ of $\mathbb{C}\left[t_{2}, t_{2}^{-1}\right]$ such that $\lambda\left(\alpha_{i}^{\vee} \otimes \mathcal{S}\right)=0$ for all $i=1,2, \ldots, s$. By the definition of $L(\lambda)$, we have $x_{\alpha_{i}} \otimes \mathcal{S} v_{\lambda}=0$ for each $i=0,1,2, \ldots, s$. The next step is to show $y_{\alpha_{i}} \otimes \mathcal{S}$. $v_{\lambda}=0$ for $i=0,1,2, \ldots, s$. Since $x_{\alpha_{j}} \otimes t_{2}^{m} \cdot y_{\alpha_{i}} \otimes \mathcal{S} \cdot v_{\lambda}=\delta_{j i} \alpha_{i}^{\vee} \otimes \mathcal{S} \cdot v_{\lambda}=0$ for arbitrary $j, i=0,1,2, \ldots, s, m \in \mathbb{Z}$ (where we set $\alpha_{0}^{\vee}=-\theta^{\vee}$ ), and $L(\lambda)$ is irreducible, one sees that $y_{\alpha_{i}} \otimes \mathcal{S} . v_{\lambda}=0$ for $i=0,1,2, \ldots, s$. Hence $\widetilde{\mathfrak{g}}_{1} \otimes \mathcal{S}$. $v_{\lambda}=0$ by induction.

Now consider $\bar{W}=\left\{w \in L(\lambda), \widetilde{\mathfrak{g}}_{1} \otimes \mathcal{S} . w=0\right\}$, which is a submodule of $L(\lambda)$. Then $L(\lambda)=\bar{W}$ by the irreducibility of $L(\lambda)$, therefore $\widetilde{\mathfrak{g}}_{1} \otimes \mathcal{S} \cdot L(\lambda)=$ 0 .

Since $\lambda\left(c_{1}\right)=\lambda\left(c_{2}\right)=0, L(\lambda)$ can be viewed as a module for the loop algebra $\mathfrak{g} \otimes \mathbb{C}\left[t_{1}, t_{1}^{-1}, t_{2}, t_{2}^{-1}\right]$. The following proposition is easily derived from Corollary [5.11] and [6, Prop. 3.8].

Proposition 5.5. Let $\mathcal{S}_{1}, \mathcal{S}_{2}$ be co-prime and co-finite ideals of $\mathbb{C}\left[t_{2}, t_{2}^{-1}\right]$, and suppose that $\lambda$ and $\mu$ satisfy the conditions in Theorem 5.7. Then $L(\lambda+\mu) \cong L(\lambda) \otimes L(\mu)$.

Rao [15] and Chang-Tan [1 have shown respectively that irreducible integrable modules for toroidal and TKK modules with finite dimensional weight spaces and $c_{1}>0, c_{2}=0$ are highest weight modules. In general our modules do not seem to be of highest weight type when $c_{1}=c_{2}=0$.

\section{Acknowledgments}

NJ acknowledges the partial support of Simons Foundation grant 198129, NSFC grant 11271138 and hospitality of Max-Planck Institute for Mathematics in the Sciences at Leipzig during this work.

\section{References}

[1] X. Chang, S. Tan, A class of irreducible integarable modules for the extended baby TKK algebra, Pacific J. Math. 252 (2011), 293-312. 
[2] V. Chari, Representations of affine and toroidal Lie algebras. Geometric representation theory and extended affine Lie algebras, 169197, Fields Inst. Commun., 59, Amer. Math. Soc., Providence, RI, 2011.

[3] V. Chari, T. Le, Representations of double affine Lie algebras, in: Trends Math, pp. 199-219, Birkhäuser, Basel, 2003.

[4] V. Chari, A. Pressley, Weyl modules for classical and quantum affine algebras, Represent. Theory 5 (2001), 191-223.

[5] M. Dokuchaev, L. V. Figueiredo, V. Futorny, Imaginary Verma modules for the extended affine Lie algebra sl $\left.\mathbb{C}_{q}\right)$, Comm. Algebra 31 (2003), 289-308.

[6] S. Eswara Rao, V. Futorny, Representations of loop Kac-Moody Lie algebras, Comm. Algebra 41 (2013), 3775-3792.

[7] I. Frenkel, Representations of Kac-Moody algebras and dual resonance models. In: Applications of group theory in physics and mathematical physics (Chicago, 1982), pp. 325-353, Lectures in Appl. Math., 21, Amer. Math. Soc., Providence, RI, 1985.

[8] V. E. Futorny, Imaginary Verma modules for affine Lie algebras. Canad. Math. Bull. 37 (1994), 213-218.

[9] V. Futorny, I. Kashuba, Verma type modules for toroidal Lie algebras, Comm. Algebra 27 (1999), 3979-3991.

[10] H. Garland, The arithmetic theory of loop algebras, J. Algebra 53 (1978), 480-551.

[11] V. G. Kac, Infinite dimensional Lie algebras, 3rd ed., Cambridge University Press, 1990.

[12] R. V. Moody, Z. Shi, Toroidal Weyl groups, Nova J. Alg. Geom. 1 (1992), 317-337.

[13] R. V. Moody, A. Pianzona, Lie algebras with triangular decompositions, John Wiley \& Sons, Inc., New York, 1995.

[14] S. E. Rao, Classification of irreducible integrable modules for multi-loop algebras with finite dimensional weight spaces, J. Algebra 246 (2001), 215-225.

[15] S. E. Rao, Classifcation of irreducible integrable modules for toroidal Lie algebras with finite dimensional weight spaces, J. Algebra 277 (2004), 318-348.

School of Mathematical Sciences, South China University of Technology, Guangzhou, Guangdong 510640, China and Department of Mathematics, North Carolina State University, Raleigh, NC 27695, USA

E-mail address: jing@math.ncsu.edu

School of Mathematical Sciences, South China University of Technology, Guangzhou, Guangdong 510640, China

E-mail address: tiankon_g1987@163.com 